\title{
MicroRNA-based molecular classification of papillary thyroid carcinoma
}

\author{
FRANCESCA ROSIGNOLO ${ }^{1}$, LORENZO MEMEO $^{2}$, FABIO MONZANI $^{3}$, CRISTINA COLAROSSI $^{2}$, \\ VALERIA PECCE $^{1}$, ANTONELLA VERRIENTI ${ }^{1}$, COSIMO DURANTE $^{1}$, GIORGIO GRANI ${ }^{1}$, \\ LIVIA LAMARTINA ${ }^{1}$, STEFANO FORTE ${ }^{4}$, DANIELA MARTINETTII ${ }^{4}$, DARIO GIUFFRIDA ${ }^{2}$, \\ DIEGO RUSSO $^{5}$, FULVIO BASOLO ${ }^{6}$, SEBASTIANO FILETTI ${ }^{1}$ and MARIALUISA SPONZIELLO ${ }^{1}$ \\ ${ }^{1}$ Department of Internal Medicine and Medical Specialties, 'Sapienza' University of Rome, Rome; \\ ${ }^{2}$ Department of Experimental Oncology, Mediterranean Institute of Oncology, Viagrande; \\ ${ }^{3}$ Department of Clinical and Experimental Medicine, University of Pisa, Pisa; ${ }^{4}$ IOM Ricerca, \\ Viagrande; ${ }^{5}$ Department of Health Sciences, University of Catanzaro 'Magna Graecia', \\ Catanzaro; ${ }^{6}$ Department of Histopathology, University of Pisa, Pisa, Italy
}

Received December 9, 2016; Accepted February 28, 2017

DOI: 10.3892/ijo.2017.3960

\begin{abstract}
MicroRNA (miRNA) expression is dysregulated in many human malignancies, and a growing number of studies are focused on their potential use as tumor biomarkers. To identify a miRNA signature for papillary thyroid carcinomas (PTC), we investigated miRNA expression profiles in two independent cohorts of PTCs, which included major histological subtypes [classical-type (PTC-CT), follicular-variant (PTC-FV), and tall-cell variant (PTC-TCV)] and cases with low or intermediate risk of recurrence. Using TaqMan ${ }^{\circledR}$ Array Human MicroRNA $\mathrm{A}+\mathrm{B}$ Cards v3.0, we first performed microRNA profiling of normal and neoplastic thyroid tissues from 29 PTC patients. Promising candidates were then investigated in a second, independent cohort of 76 PTCs using Custom TaqMan ${ }^{\circledR}$ Array MicroRNA Cards. We identified a molecular signature of 11 miRNAs that were significantly upregulated (miR-146b-5p, miR-146b-3p, miR-221-3p, miR-222-5p, miR-222-3p) or downregulated (miR-1179, miR-486-5p, miR-204-5p, miR-7-2-3p, miR-144-5p, miR-140-3p) in PTC tissues vs. normal thyroid tissue. Upregulation of miR-146b-5p and miR-222-3p was also significantly associated with an increased risk of recurrence. Higher than normal expression of miR-146b-5p and miR-146b-3p characterized PTC-CT and PTC-TCV but not
\end{abstract}

Correspondence to: Dr Marialuisa Sponziello, Department of Internal Medicine and Medical Specialties, 'Sapienza' University of Rome, Viale del Policlinico 155, I-00161 Rome, Italy

E-mail: marialuisa.sponziello@uniroma1.it

Abbreviations: miRNA, microRNA; PTC, papillary thyroid carcinoma; PTC-CT, classical type; PTC-FV, follicular variant; PTC-TCV, tall cell variant; PTC-EFV, encapsulated follicular variant; PTC-IFV, infiltrative follicular variant

Key words: miRNA, papillary thyroid carcinoma, histotypes, recurrences
PTC-FV, whereas miR-21-5p was significantly upregulated only in PTC-TCV. When PTC-FV were subclassified as encapsulated (PTC-EFV) or infiltrative (PTC-IFV), miR-204-5p was downregulated in all histological subtypes except PTC-EFV, which displayed expression levels similar to those of normal thyroid tissues. These findings provide new insights into the molecular classification of PTC, showing that different miRNA expression profiles are associated with different histological types of PTC and different risks of recurrence.

\section{Introduction}

Papillary thyroid carcinoma (PTC) accounts for $\sim 85 \%$ of all well-differentiated thyroid cancers and is thus the most common thyroid malignancy $(1,2)$. Most PTCs are small tumors with limited extension, indolent growth, and excellent prognoses, but $\sim 18 \%$ exhibit aggressive clinical behavior (2). PTCs include several histological types: classical forms (PTC-CT), which are the most common, and follicular- and tall-cell variants (PTC-FV and PTC-TC, respectively). Each histotype is characterized by specific clinicopathological features (3). PTC-TC is the most aggressive of the three, while PTC-FV is the most indolent (4).

The generally non-aggressive clinical behavior of PTC is consistent with its genetic and biologic characteristics. The mutation density of the PTC genome is on the whole lower than that of other cancers, which reflects its indolent behavior $(5,6)$. In addition, thyroid differentiation score (based on expression level of thyroid metabolism and function genes) correlates with histological grade, risk of recurrence and mortality of PTCs (5). Greater understanding of the molecular underpinning of thyroid cancers will necessarily improve their diagnosis and treatment, especially for certain subtypes whose classification criteria are less rigorously defined and objectively debatable $(7,8)$.

In some studies, tumor miRNA profiles have proven to be more useful for classifying cancers than sequencing analysis or gene expression profiling (9). Their high stability 
in paraffin-embedded tissues (10) and body fluids (11) makes miRNAs excellent candidates as biomarkers for many cancers, including PTC $(5,12)$. Several studies have explored the expression profiles of these non-coding RNA species in PTCs, and hundreds of miRNAs reportedly display tumorrelated dysregulation in PTCs (5,13-15). However, the specific miRNAs identified as dysregulated vary from study to study, and the results are often discordant (16). Less is known about the association between miRNA expression and the clinicopathological features of PTC, such as clinical aggressiveness and histological features $(17,18)$.

The aim of this study was to identify miRNAs with dysregulated expression in PTC, with particular emphasis on alterations associated with specific histological types and/or with the risk of tumor recurrence, in order to clarify the role of miRNAs as effective biomarkers for tumor classification.

\section{Materials and methods}

Study design and patient samples. The study was conducted with institutional review board approval and the written informed consent of all patients whose tissues were analyzed. Our primary aim was to define a microRNA signature for sporadic PTC tissue. To this end, we enrolled two independent cohorts of patients with sporadic PTCs who underwent thyroidectomy between 2012 and 2014 at the Department of Internal Medicine and Medical Specialties of 'Sapienza' University of Rome or at the Department of Surgical Pathology of the University of Pisa.

Immediately after surgery, samples of tumor tissue and normal tissue from the unaffected lobe were collected prospectively from each participant of cohort I. Tissues were snap-frozen and stored in liquid nitrogen prior to microRNA profiling analysis (described below). On the basis of the results of the screening analysis and a review of the literature, we selected a panel of miRNAs for further validation. Their expression was evaluated in formalin-fixed, paraffin-embedded (FFPE) samples of normal and neoplastic thyroid tissues from the patients making up cohort II.

The secondary aims of the study were to identify miRNAs whose dysregulated expression was associated with one or more histological types of PTC and/or with an elevated risk of posttreatment recurrence. These issues were explored in cohort II, where enrolled cases had been selected specifically to ensure roughly equal representation of low- and intermediate-risk cases, as defined by the American Thyroid Association (ATA) (19), and the maximum number possible of histological PTC variants (based on availability).

Fresh-frozen and FFPE tissue samples were reviewed separately by two pathologists, who confirmed the diagnosis of PTC, identified the tumor histotype, excluded fresh frozen tumor samples in which tumor cells accounted for $<60 \%$ of the total, and marked tumor tissue in each slide for macrodissection.

Analysis of tissue miRNAs. Total RNA containing small RNAs was extracted from fresh-frozen tissues (cohort I) using TRIzol reagent (Thermo Fisher Scientific, Inc., Waltham, MA, USA) and from FFPE tissues (cohort II) using the mirVana ${ }^{\mathrm{TM}}$ miRNA Isolation kit (Thermo Fisher Scientific). The quality
Table I. Baseline characteristics of PTC patients in the study cohorts.

\begin{tabular}{|c|c|c|}
\hline $\begin{array}{l}\text { Clinicopathological } \\
\text { features - } \mathrm{n}(\%)\end{array}$ & $\begin{array}{l}\text { Cohort I } \\
(n=29)\end{array}$ & $\begin{array}{c}\text { Cohort II } \\
(\mathrm{n}=76)\end{array}$ \\
\hline \multicolumn{3}{|c|}{ Age at diagnosis (years) ${ }^{\mathrm{a}}$} \\
\hline$<45$ & $6(20.7)$ & $39(51.3)$ \\
\hline$\geq 45$ & $23(79.3)$ & $36(47.4)$ \\
\hline \multicolumn{3}{|l|}{ Gender } \\
\hline Male & $8(27.6)$ & $19(25.0)$ \\
\hline Female & $21(72.4)$ & $57(75.0)$ \\
\hline \multicolumn{3}{|l|}{ Tumor size $(\mathrm{cm})$} \\
\hline$\geq 1$ & $20(69.0)$ & $48(63.2)$ \\
\hline$<1$ & $9(31.0)$ & $28(36.8)$ \\
\hline \multicolumn{3}{|l|}{ Multifocality } \\
\hline Yes & $9(31.0)$ & $28(36.8)$ \\
\hline No & $20(69.0)$ & $48(63.2)$ \\
\hline \multicolumn{3}{|c|}{ Extrathyroidal extension } \\
\hline Yes & $11(37.9)$ & $29(38.2)$ \\
\hline No & $18(62.1)$ & $47(61.8)$ \\
\hline \multicolumn{3}{|c|}{ Lymph node metastases } \\
\hline Yes & $8(27.6)$ & $26(34.2)$ \\
\hline No & $21(72.4)$ & $50(65.8)$ \\
\hline \multicolumn{3}{|l|}{ ATA risk 2015} \\
\hline Low & $10(34.5)$ & $33(43.4)$ \\
\hline Intermediate & $19(65.5)$ & $43(56.6)$ \\
\hline \multicolumn{3}{|l|}{ Histological variant } \\
\hline PTC-CT & $23(79.3)$ & $47(61.8)$ \\
\hline PTC-FV & $5(17.2)$ & $20(26.3)$ \\
\hline PTC-EFV & $3(10.3)$ & $14(18.4)$ \\
\hline PTC-IFV & $2(6.9)$ & $6(7.9)$ \\
\hline PTC-TCV & 0 & $7 \quad(9.2)$ \\
\hline Other & $1(3.4)$ & $2(2.6)$ \\
\hline
\end{tabular}

ATA, American Thyroid Association; PTC-CT, classical variant; PTC-FV, follicular variant; PTC-TCV, tall cell variant; PTC-EFV, encapsulated follicular variant; PTC-IFV, infiltrative follicular variant. ${ }^{\text {aD }}$ ata unavailable for one patient.

and quantity of RNA samples were verified with a NanoDrop spectrophotometer (Thermo Fisher Scientific).

The screening analysis consisted of miRNA profiling performed on fresh-frozen thyroid tissues from cohort I PTC patients. TaqMan Array Human MicroRNA A+B Cards v3.0 (Thermo Fisher Scientific), a set of two 384-well microfluidic cards, were used to quantify the relative expression of 754 miRNAs as previously reported (20). In the validation analysis, we evaluated the expression of a selected panel of miRNAs in FFPE samples of thyroid tissue from cohort II PTC patients using Custom TaqMan Array MicroRNA Cards (Thermo Fisher Scientific). Each array was configured with specific TaqMan miRNA expression assays (Thermo Fisher Scientific). In both analyses, TaqMan arrays were processed as previously reported (21). Expression Suite software v1.0.3 (Thermo Fisher Scientific) was used to calculate $\mathrm{Ct}$ values 


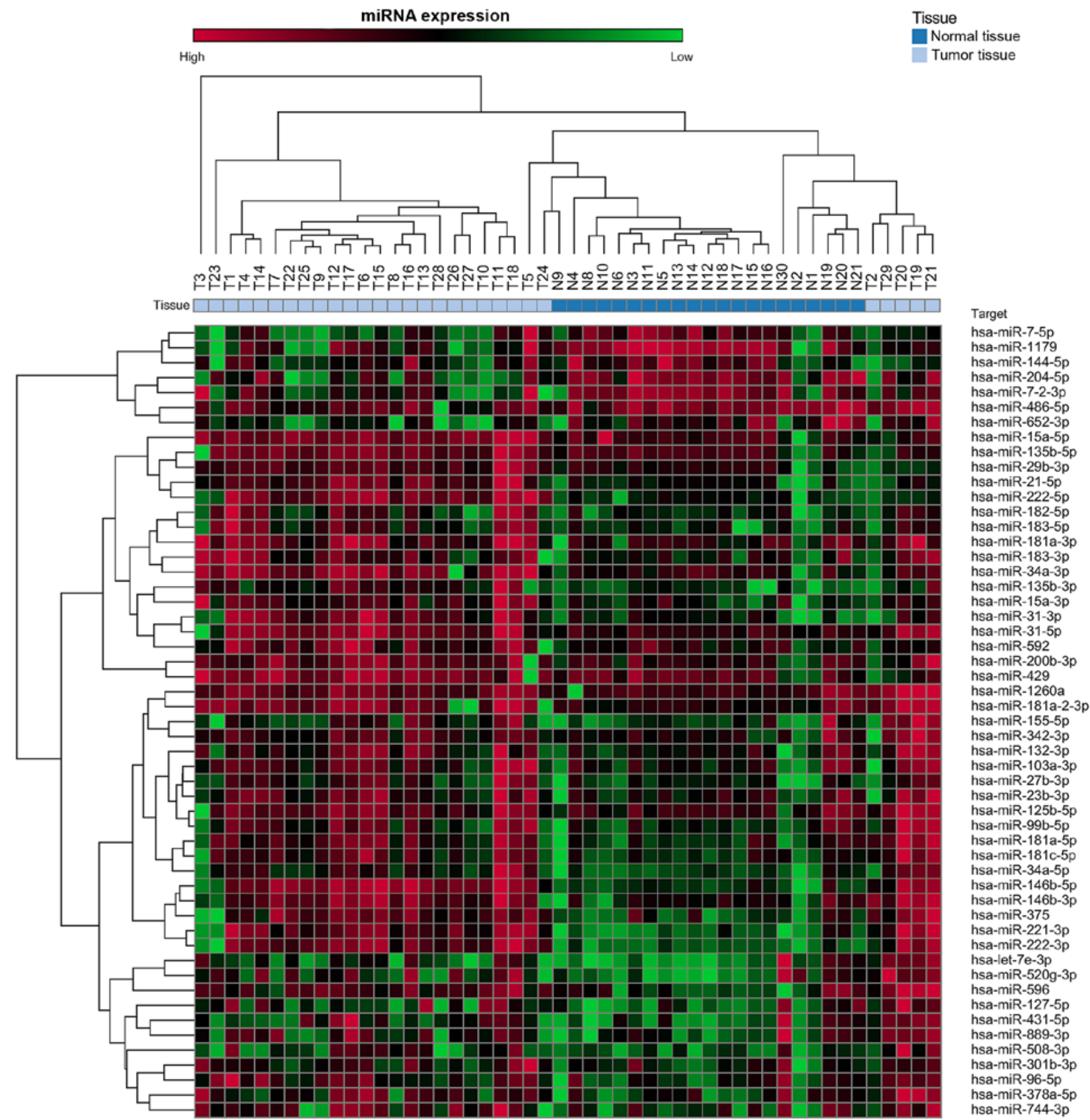

Figure 1. Heat map and hierarchical clustering of miRNA profiling. The columns represent the samples and the rows represent the miRNAs. The scale color from red (high expression) to green (low expression) reports the expression levels of each miRNA (expressed as $\Delta \mathrm{CT}$ value normalized by using U6 as endogenous control).

and relative miRNA expression (using the comparative $2^{-\Delta \Delta C t}$ method). The Ct cut-off was set at 35 , and U6 was used as an endogenous control.

Statistical analysis. Differences between two groups were assessed with the Mann-Whitney U test followed by either Benjamini-Hochberg correction (false discovery rate, FDR) (in the screening analysis) or Bonferroni correction (in the validation analysis). When three or more groups were compared, differences were assessed with the Kruskal-Wallis test followed by the post hoc Dunn's multiple comparison test. The Mann-Whitney and Kruskall-Wallis tests were carried out using SPSS software version 22.0 (IBM Corp., Armonk, NY, USA). The 'p.adjust' function of the basic R stats package (R software version 3.1.1) $(22,23)$ was used for Benjamini-Hochberg and Bonferroni corrections. Heat maps and hierarchical clustering based on $\Delta \mathrm{Ct}$ values were done with GENE-E software version 3.0.230 (http://www. broadinstitute.org/cancer/software/GENE-E), using Spearman correlation and complete linkage.

\section{Results}

Dysregulated miRNA expression in PTC tissues

Screening analysis. Table I shows the characteristics of the 29 PTC patients enrolled in cohort I. Of the 754 miRNAs analyzed in this cohort, 53 exhibited mean levels in the tumor tissues that were significantly higher $(n=46)$ or lower $(n=7)$ than the means for the normal thyroid tissues (Fig. 1 and Table II). 
Table II. MicroRNAs displaying dysregulated expression in tumor tissues of PTC patients from the cohort I.

\begin{tabular}{|c|c|c|c|c|}
\hline miRBase ID v21 & Normal tissues $(n=21)$ & Tumor tissues $(\mathrm{n}=29)$ & P-value & P-value adj \\
\hline hsa-miR-146b-5p & $1(0.018-7.340)$ & $58.758(0.094-230.834)$ & $<0.0001$ & 0.0036 \\
\hline hsa-miR-221-3p & $1(0.095-4.860)$ & $24.204(0.233-97.736)$ & $<0.0001$ & 0.0036 \\
\hline hsa-miR-222-3p & $1(0.260-2.785)$ & $17.707(0.201-61.485)$ & $<0.0001$ & 0.0036 \\
\hline hsa-miR-222-5p & $1(0.039-2.351)$ & $15.973(0.157-66.455)$ & $<0.0001$ & 0.0036 \\
\hline hsa-miR-146b-3p & $1(0.005-6.837)$ & $10.861(0.017-55.237)$ & $<0.0001$ & 0.0036 \\
\hline hsa-miR-34a-5p & $1(0.083-4.066)$ & $9.275(0.257-88.148)$ & $<0.0001$ & 0.0036 \\
\hline hsa-miR-31-3p & $1(0.047-2.772)$ & $8.736(0.066-38.979)$ & $<0.0001$ & 0.0036 \\
\hline hsa-miR-21-5p & $1(0.028-2.803)$ & $8.706(0.119-55.252)$ & $<0.0001$ & 0.0036 \\
\hline hsa-miR-375 & $1(0.066-6.745)$ & $7.764(0.034-44.529)$ & $<0.0001$ & 0.0036 \\
\hline hsa-miR-31-5p & $1(0.323-2.424)$ & $7.264(0.004-29.557)$ & $<0.0001$ & 0.0036 \\
\hline hsa-miR-135b-3p & $1(0.121-2.507)$ & $6.102(0.224-51.519)$ & $<0.0001$ & 0.0036 \\
\hline hsa-miR-182-5p & $1(0.063-3.459)$ & $5.780(0.099-38.691)$ & 0.0163 & NS \\
\hline hsa-miR-508-3p & $1(0.137-6.107)$ & $5.249(0.094-52.703)$ & 0.0252 & NS \\
\hline hsa-miR-181a-2-3p & $1(0.267-4.857)$ & $4.886(0.009-16.393)$ & $<0.0001$ & 0.0036 \\
\hline hsa-miR-34a-3p & $1(0.031-2.483)$ & $4.836(0.022-14.827)$ & $<0.0001$ & 0.0036 \\
\hline hsa-miR-183-5p & $1(0.074-2.680)$ & $4.518(0.157-28.343)$ & 0.0021 & NS \\
\hline hsa-miR-1260a & $1(0.000-6.705)$ & $4.292(0.366-23.045)$ & 0.0005 & 0.0164 \\
\hline hsa-miR-183-3p & $1(0.055-7.218)$ & $4.027(0.027-19.837)$ & 0.0013 & 0.0392 \\
\hline hsa-miR-181a-3p & $1(0.014-4.661)$ & $3.916(0.154-12.278)$ & 0.0052 & NS \\
\hline hsa-miR-15a-3p & $1(0.078-3.530)$ & $3.638(0.313-16.829)$ & $<0.0001$ & 0.0036 \\
\hline hsa-miR-29b-3p & $1(0.013-2.231)$ & $3.631(0.052-31.111)$ & 0.0055 & NS \\
\hline hsa-miR-181a-5p & $1(0.115-2.623)$ & $3.301(0.321-11.416)$ & $<0.0001$ & 0.0036 \\
\hline hsa-miR-181c-5p & $1(0.146-2.233)$ & $3.192(0.217-12.265)$ & $<0.0001$ & 0.0036 \\
\hline hsa-miR-378a-5p & $1(0.052-6.176)$ & $3.011(0.114-14.683)$ & 0.0040 & NS \\
\hline hsa-miR-596 & $1(0.060-5.028)$ & $2.965(0.289-11.180)$ & $<0.0001$ & 0.0036 \\
\hline hsa-miR-27b-3p & $1(0.156-2.139)$ & $2.565(0.302-13.316)$ & 0.0067 & NS \\
\hline hsa-miR-592 & $1(0.133-3.765)$ & $2.548(0.032-11.515)$ & 0.0343 & NS \\
\hline hsa-miR-744-3p & $1(0.080-3.584)$ & $2.438(0.069-10.357)$ & 0.0122 & NS \\
\hline hsa-miR-127-5p & $1(0.072-6.326)$ & $2.430(0.098-16.629)$ & 0.0280 & $\mathrm{NS}$ \\
\hline hsa-miR-135b-5p & $1(0.023-2.518)$ & $2.357(0.009-12.712)$ & 0.0115 & NS \\
\hline hsa-miR-155-5p & $1(0.083-10.923)$ & $2.320(0.059-15.242)$ & 0.0343 & NS \\
\hline hsa-miR-96-5p & $1(0.054-5.897)$ & $2.294(0.283-12.549)$ & 0.0063 & NS \\
\hline hsa-miR-15a-5p & $1(0.002-10.510)$ & $2.179(0.043-8.537)$ & 0.0001 & 0.0036 \\
\hline hsa-miR-99b-5p & $1(0.162-2.991)$ & $2.173(0.340-6.729)$ & 0.0115 & NS \\
\hline hsa-miR-23b-3p & $1(0.083-3.789)$ & $2.165(0.092-7.254)$ & 0.0239 & NS \\
\hline hsa-miR-520g-3p & $1(0.126-7.794)$ & $2.100(0.149-9.733)$ & 0.0052 & NS \\
\hline hsa-miR-429 & $1(0.036-3.551)$ & $2.088(0.008-6.988)$ & 0.0215 & NS \\
\hline hsa-miR-125b-5p & $1(0.070-3.085)$ & $2.069(0.021-7.428)$ & 0.0122 & NS \\
\hline hsa-miR-200b-3p & $1(0.135-2.647)$ & $2.052(0.052-6.990)$ & 0.0042 & NS \\
\hline hsa-miR-132-3p & $1(0.115-3.208)$ & $2.045(0.268-6.510)$ & 0.0145 & NS \\
\hline hsa-miR-431-5p & $1(0.099-8.621)$ & $1.839(0.165-10.632)$ & 0.0085 & NS \\
\hline hsa-miR-103a-3p & $1(0.206-2.820)$ & $1.755(0.138-4.958)$ & 0.0154 & NS \\
\hline hsa-miR-301b-3p & $1(0.100-4.459)$ & $1.752(0.340-9.937)$ & 0.0115 & NS \\
\hline hsa-miR-342-3p & $1(0.197-4.199)$ & $1.617(0.097-5.774)$ & 0.0109 & NS \\
\hline hsa-miR-889-3p & $1(0.092-7.992)$ & $1.573(0.123-5.696)$ & 0.0265 & NS \\
\hline hsa-let-7e-3p & $1(0.145-9.472)$ & $1.383(0.153-5.515)$ & 0.0145 & NS \\
\hline hsa-miR-7-2-3p & $1(0.008-2.544)$ & $0.446(0.003-4.165)$ & 0.0005 & 0.0164 \\
\hline hsa-miR-652-3p & $1(0.081-3.271)$ & $0.441(0.072-2.357)$ & 0.0043 & NS \\
\hline hsa-miR-486-5p & $1(0.010-4.845)$ & $0.406(0.000-2.334)$ & 0.0043 & NS \\
\hline hsa-miR-7-5p & $1(0.022-4.102)$ & $0.378(0.010-5.822)$ & 0.0006 & 0.0189 \\
\hline hsa-miR-204-5p & $1(0.061-2.609)$ & $0.346(0.019-1.560)$ & $<0.0001$ & 0.0036 \\
\hline hsa-miR-144-5p & $1(0.014-5.825)$ & $0.339(0.005-2.837)$ & 0.0173 & NS \\
\hline hsa-miR-1179 & $1(0.010-2.048)$ & $0.255(0.010-2.025)$ & $<0.0001$ & 0.0036 \\
\hline
\end{tabular}

Relative miRNA expression levels for tumor tissues are reported as means (minimum-maximum) normalized to those for normal tissues (equal to 1).

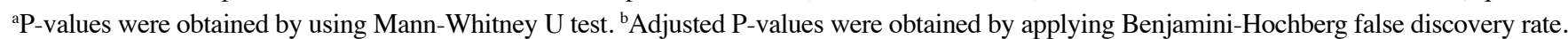


Table III. The 30 miRNAs selected for the validation analysis.

\begin{tabular}{|c|c|}
\hline miRBase ID v21 & miR assay ID \\
\hline hsa-miR-1179 & hsa-miR-1179-002776 \\
\hline hsa-miR-146b-5p & hsa-miR-146b-001097 \\
\hline hsa-miR-146b-3p & hsa-miR-146b-3p-002361 \\
\hline hsa-miR-15a-3p & hsa-miR-15a*-002419 \\
\hline hsa-miR-181a-5p & hsa-miR-181a-000480 \\
\hline hsa-miR-181a-2-3p & hsa-miR-181a-2*-002317 \\
\hline hsa-miR-183-5p & hsa-miR-183-002269 \\
\hline hsa-miR-204-5p & hsa-miR-204-000508 \\
\hline hsa-miR-21-5p & hsa-miR-21-000397 \\
\hline hsa-miR-181a-3p & hsa-miR-213-000516 \\
\hline hsa-miR-221-3p & hsa-miR-221-000524 \\
\hline hsa-miR-222-5p & hsa-miR-222*-002097 \\
\hline hsa-miR-222-3p & hsa-miR-222-002276 \\
\hline hsa-miR-31-5p & hsa-miR-31-002279 \\
\hline hsa-miR-34a-3p & hsa-miR-34a* -002316 \\
\hline hsa-miR-34a-5p & hsa-miR-34a-000426 \\
\hline hsa-miR-375 & hsa-miR-375-000564 \\
\hline hsa-miR-486-5p & hsa-miR-486-001278 \\
\hline hsa-miR-652-3p & hsa-miR-652-002352 \\
\hline hsa-miR-7-2-3p & hsa-miR-7-2*-002314 \\
\hline hsa-miR-144-5p & hsa-miR-144*-002148 \\
\hline hsa-miR-182-5p & hsa-miR-182-002334 \\
\hline hsa-miR-103a-3p & hsa-miR-103-000439 \\
\hline hsa-miR-125b-5p & hsa-miR-125b-000449 \\
\hline hsa-miR-135b-5p & hsa-miR-135b-002261 \\
\hline hsa-miR-200b-3p & hsa-miR-200b-002251 \\
\hline hsa-miR-155-5p & hsa-miR-155-002623 \\
\hline hsa-miR-1908-5p ${ }^{a}$ & hsa-miR-1908-121109_mat \\
\hline hsa-miR-140-3p ${ }^{b}$ & hsa-miR-140-3p-002234 \\
\hline hsa-miR-199b-3p/ & hsa-miR-199a-3p-002304 \\
\hline hsa-miR-199a-3p $p^{b}$ & \\
\hline
\end{tabular}

${ }^{a}$ Not included in the TaqMan Array Human MicroRNA A+B Cards v3.0 (Thermo Fisher Scientific). ${ }^{b}$ Not significantly dysregulated in the tumor tissues of cohort I PTCs.

Validation analysis. From this set of 53 miRNAs, we selected a panel of 30 miRNAs for validation in the 76 patients making up cohort II (Table I). It included 27 miRNAs with markedly dysregulated expression documented in the tumor tissues of cohort I PTCs as well as in PTC tissues studied by other groups $(5,13-15)$ and three other miRNAs PTC- or cancer-related $(5,24-28)$, which were either not analyzed in the screening analysis or not significantly dysregulated in cohort I (Table III). The results of this analysis identified a signature of 11 miRNAs (miR-146b-5p, miR-146b-3p, miR-221-3p, miR-222-5p, miR-222-3p, miR-1179, miR-486-5p, miR-204-5p, miR-7-2-3p, miR-144-5p, miR-140-3p) that were significantly dysregulated in PTC tumor tissues, as compared with normal

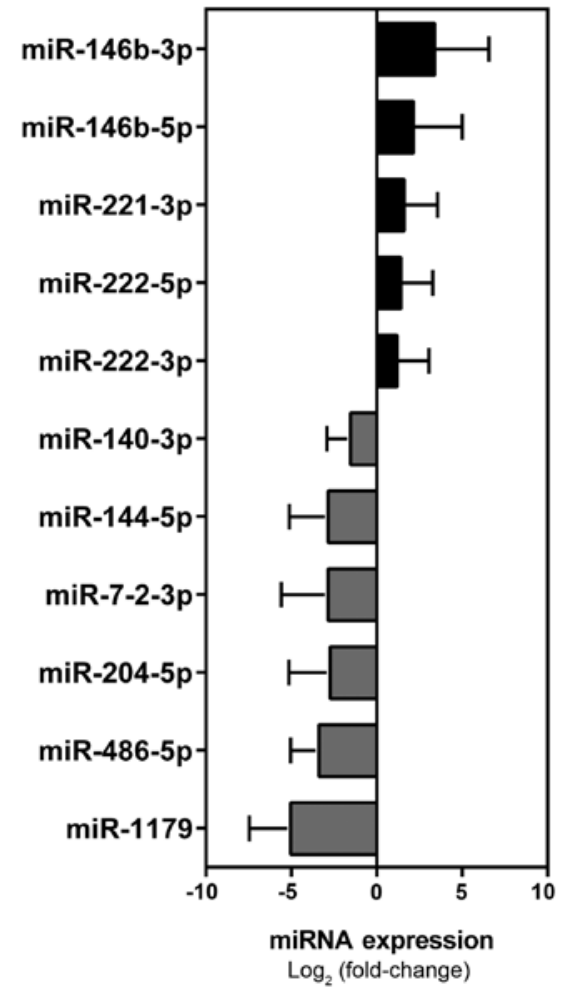

Figure 2. Validation of miRNAs significantly dysregulated in tumor tissues of PTC from cohort II. Relative expression levels of each miRNA are reported as mean \pm SD normalized to mean expression levels in normal thyroid tissues (equal to 1). Black and grey bars indicate upregulated and downregulated miRNAs in tumor tissues, respectively.

tissues from the unaffected lobe (Mann-Whitney followed by Bonferroni correction) (Table IV and Fig. 2).

Dysregulated miRNA expression in PTC histotypes. Next, we re-analyzed the expression of the 30 miRNAs listed in Table III as a function of PTC histotype. This analysis was restricted to the 74 cohort II PTCs representing the three main histotypes (PTC-CT, PTC-FV, PTC-TCV). The remaining two cases in cohort II were excluded, because they were rare PTC variants (trabecular in one case, sclerosing in the other). The results of this analysis are summarized in Table V. Overall, expression levels of 13 miRNAs were significantly different among PTC-CT, PTC-FV, PTC-TCV and normal thyroid tissues (Kruskall-Wallis). Pair-wise comparisons (post hoc Dunn's test) revealed 11 miRNAs with significantly dysregulated expression (compared with that in normal thyroid tissue levels) in PTC-CT (miR-1179, miR-140-3p, miR-144-5p, miR-146b-5p, miR-146b-3p, miR-200b-3p, miR-204-5p, miR-221-3p, miR-222-5p, miR-486-5p, miR-7-2-3p). Far fewer miRNAs (miR-200b-3p, miR-221-3p, miR-486-5p) displayed altered expression in PTC-FV, which are more indolent than other PTCs. Surprisingly, the aggressive PTC-TCV was also characterized by fewer significantly dysregulated miRNAs than PTC-CT (miR-146b-5p, miR-146b-3p, miR-204-5p, miR-21-5p, miR-221-3p, miR-222-5p) (Table V). This finding might be due to the low number of samples in the PTC-TCV subgroup ( $n=7$ vs. $n=47$ in the PTC-CT group), which limited the statistical significance of several additional dysregulations observed in these tumors. 
Table IV. Dysregulated miRNAs in tumor tissues of PTC patients from cohort II.

\begin{tabular}{lcccc}
\hline miRBase ID v21 & Normal tissues $(\mathrm{n}=24)$ & Tumor tissues $(\mathrm{n}=76)$ & P-value $^{\mathrm{a}}$ & P-value adj $^{\mathrm{b}}$ \\
\hline hsa-miR-146b-5p & $1(0.074-6.969)$ & $13.58(0.024-76.340)$ & $<0.0001$ & 0.003 \\
hsa-miR-146b-3p & $1(0.130-4.807)$ & $45.890(0.048-422.9)$ & $<0.0001$ & 0.003 \\
hsa-miR-221-3p & $1(0.035-5.892)$ & $6.453(0.099-63.050)$ & $<0.0001$ & 0.003 \\
hsa-miR-222-5p & $1(0.082-3.004)$ & $5.324(0.119-29.530)$ & $<0.0001$ & 0.003 \\
hsa-miR-222-3p & $1(0.088-2.426)$ & $4.421(0.049-35.360)$ & $<0.0001$ & 0.003 \\
hsa-miR-375 & $1(0.043-2.866)$ & $3.338(0.035-31.580)$ & 0.0439 & $\mathrm{NS}$ \\
hsa-miR-21-5p & $1(0.052-4.248)$ & $2.788(0.015-29.040)$ & 0.0497 & $\mathrm{NS}$ \\
hsa-miR-31-5p & $1(0.132-4.033)$ & $2.318(0.025-8.377)$ & 0.007 & $\mathrm{NS}$ \\
hsa-miR-34a-5p & $1(0.028-3.767)$ & $2.22(0.092-15.820)$ & 0.0339 & $\mathrm{NS}$ \\
hsa-miR-34a-3p & $1(0.163-2.072)$ & $2.215(0.143-18.630)$ & 0.0376 & $\mathrm{NS}$ \\
hsa-miR-652-002352 & $1(0.115-3.188)$ & $0.6674(0.039-4.294)$ & 0.0262 & $\mathrm{NS}$ \\
hsa-miR-199a-3p/ & $1(0.067-4.098)$ & $0.5618(0.029-2.346)$ & 0.0125 & $\mathrm{NS}$ \\
hsa-miR-199b-3p & & & & $\mathrm{NS}$ \\
hsa-miR-135b-5p & $1(0.095-3.703)$ & $0.5442(0.013-3.432)$ & 0.0171 & 0.021 \\
hsa-miR-140-3p & $1(0.232-2.993)$ & $0.5089(0.017-1.752)$ & 0.0007 & 0.003 \\
hsa-miR-144-5p & $1(0.092-5.406)$ & $0.4559(0.008-3.894)$ & 0.0001 & 0.03 \\
hsa-miR-7-2-3p & $1(0.082-3.844)$ & $0.4441(0.002-3.565)$ & 0.001 & 0.027 \\
hsa-miR-204-5p & $1(0.079-3.468)$ & $0.4394(0.004-2.359)$ & 0.009 & 0.003 \\
hsa-miR-486-5p & $1(0.036-3.322)$ & $0.1874(0.009-1.298)$ & $<0.0001$ & 0.003 \\
hsa-miR-1179 & $1(0.091-6.515)$ & $0.094(0.001-0.513)$ & $<0.0001$ & \\
\hline
\end{tabular}

Relative miRNA expression levels for tumor tissues are reported as means (minimum-maximum) normalized to those for normal tissues (equal to 1).

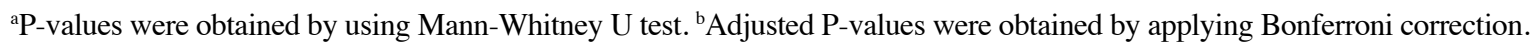

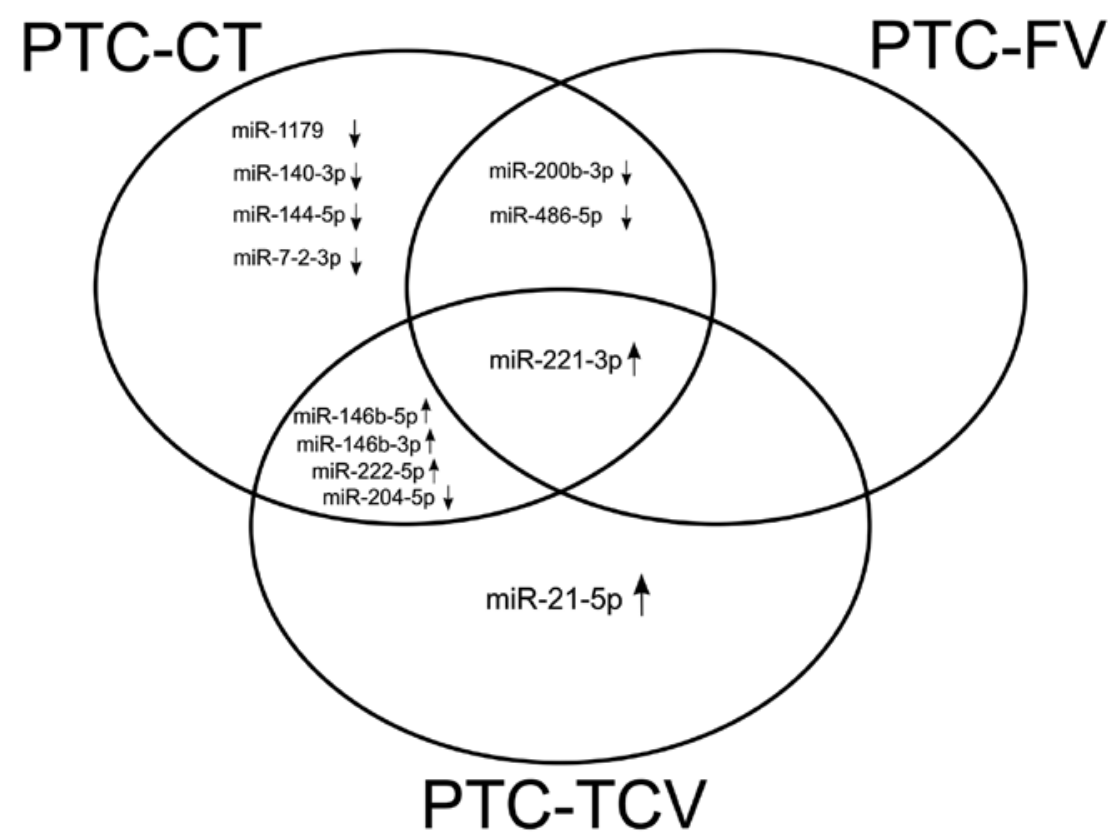

Figure 3. Venn diagram of significantly dysregulated miRNAs in PTC-CT, PTC-FV, PTC-TCV as compared with normal tissues. Significant dysregulation was defined as differential expression vs. normal thyroid tissue levels with a $\mathrm{P}_{\mathrm{adj}}<0.05$ (Kruskal-Wallis test followed by post hoc Dunn's multiple comparison test).

As shown in Fig. 3, certain dysregulation appeared to be histotype-specific, such as the significantly upregulated expression of miR-21-5p, which was found exclusively in PTC-TCV, and the significant downregulation of miR-1179, miR-140-3p, miR-144-5p and miR-7-2-3p, which appeared to be specific to PTC-CT. miR-221-3p was the only miRNA that was significantly dysregulated in all three histotypes.

Quantitatively speaking, there were no significant differences between PTC-CT and PTC-TCV in the expression of any of the miRNAs (Table V). Conversely, three miRNAs 


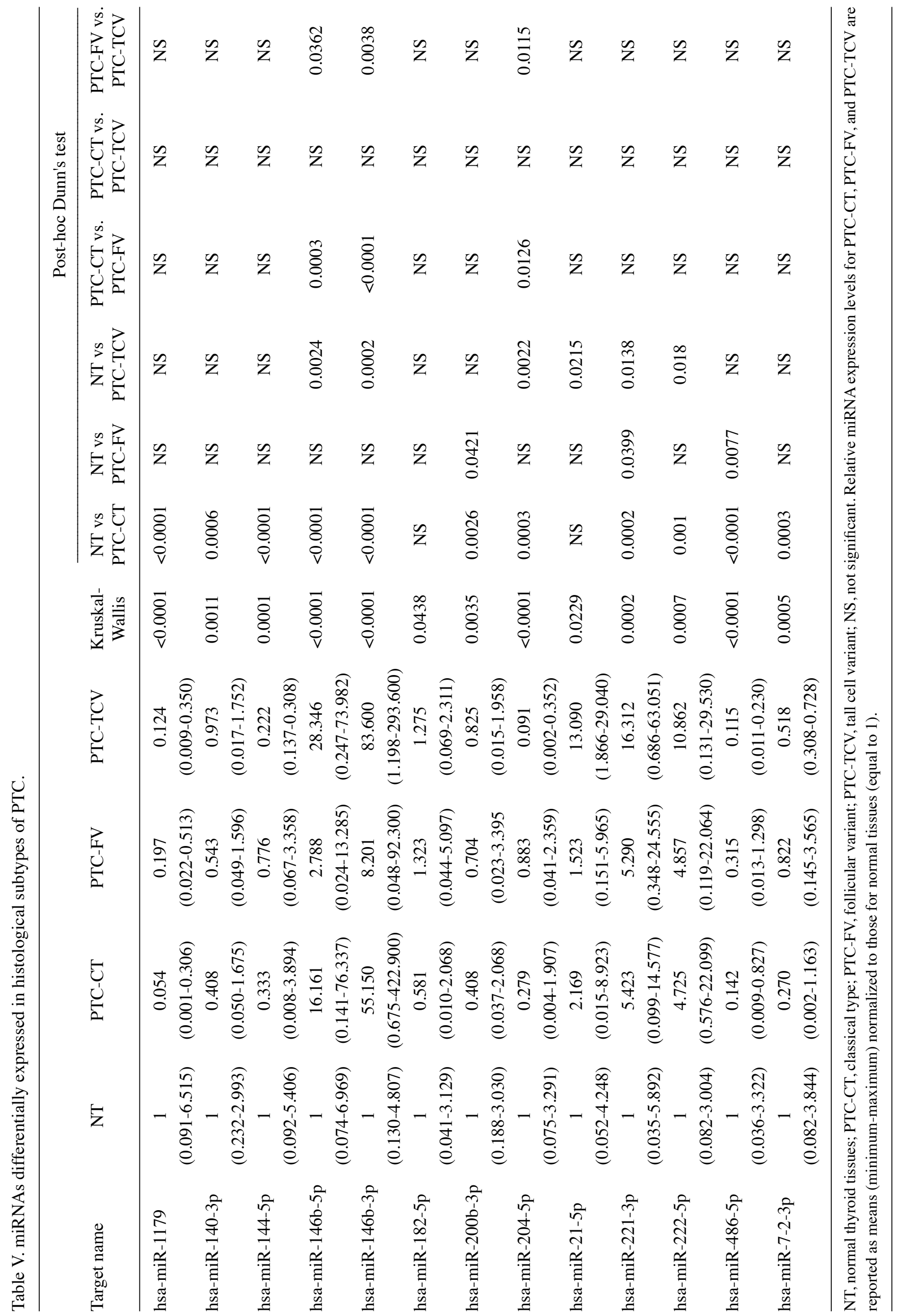


A
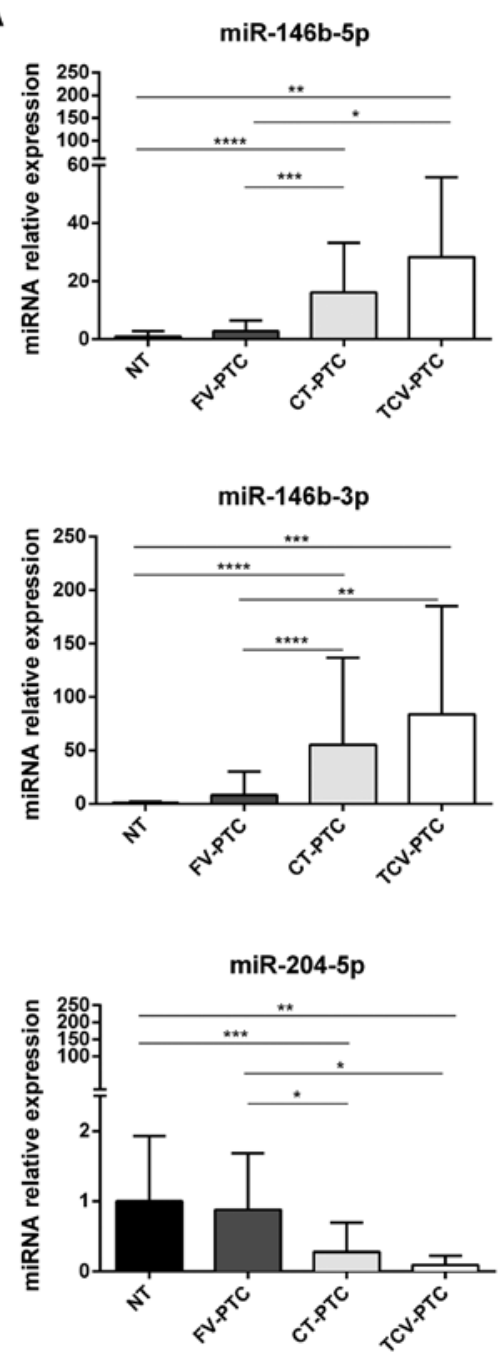

B
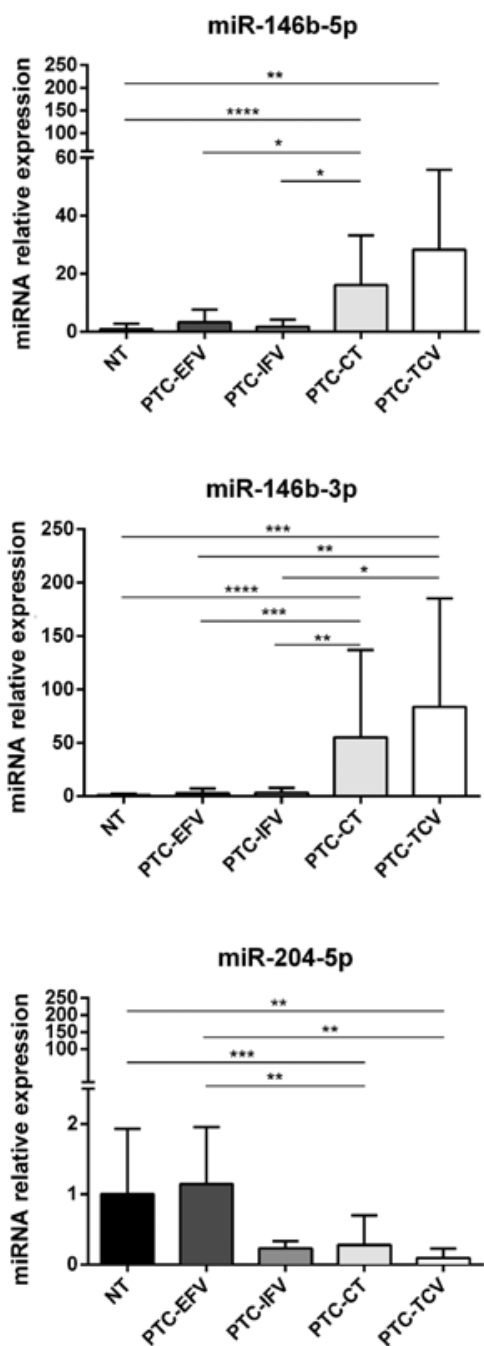

Figure 4. miRNAs differentially expressed in histological subtypes of PTC from the validation cohort. Expression of miR-146b-5p, miR-146b-3p and miR-204-5p in samples of normal thyroid tissue (NT, $n=24)$ vs. different histotypes of PTC ( $\mathrm{n}=74)$. (A) Comparison of NT levels with those found in follicular variant (PTC-FV, $n=20)$, classical type (PTC-CT, $n=47)$, and tall-cell variant (PTC-TCV, $n=7$ ); (B) Expression levels of each miRNA shown in (A) for NT, PTC-CT, and PTC-TCV are compared with those found in encapsulated and infiltrative subtypes of follicular variant PTCs [PTC-EFV (n=14) and PTC-IFV $(n=6)$, respectively]. miRNA expression levels are reported as mean expression value of each PTC variant normalized to mean expression of NT (equal to 1). Error bars represent standard deviation. P-values were obtained by using Kruskal-Wallis test followed by Dunn's multiple comparisons test: ${ }^{*} \mathrm{P}<0.05$, ${ }^{* *} \mathrm{P}<0.01$, ${ }^{* * *} \mathrm{P}<0.001$. NS, not significant.

Table VI. miRNAs that were differentially expressed in intermediate and low-risk PTCs from cohort II.

\begin{tabular}{lcccc}
\hline & Low risk $(\mathrm{n}=33)$ & Intermediate risk $(\mathrm{n}=43)$ & P-value $^{\mathrm{a}}$ & ${\text { P-value } \text { adj }^{\mathrm{b}}}$ \\
\hline hsa-miR-146b-5p & $1(0.005-8.793)$ & $4.346(0.054-16.620)$ & $<0.0001$ & 0.0030 \\
hsa-miR-21-5p & $1(0.012-4.775)$ & $3.053(0.019-23.240)$ & 0.0152 & $\mathrm{NS}$ \\
hsa-miR-222-3p & $1(0.021-6.368)$ & $2.541(0.069-15.210)$ & 0.0003 & 0.0090 \\
hsa-miR-31-5p & $1(0.184-4.029)$ & $2.192(0.019-6.297)$ & 0.0034 & $\mathrm{NS}$ \\
hsa-miR-199a-3p/ & $1(0.084-4.248)$ & $1.577(0.069-5.569)$ & 0.0131 & $\mathrm{NS}$ \\
hsa-miR-199b-3p & & & & $\mathrm{NS}$ \\
hsa-miR-146b-3p & $1(0.001-7.784)$ & $1.421(0.018-11.210)$ & 0.0077 & $\mathrm{NS}$ \\
hsa-miR-1179 & $1(0.006-3.802)$ & $0.427(0.017-2.592)$ & 0.0388 & $\mathrm{NS}$ \\
hsa-miR-7-2-3p & $1(0.003-5.557)$ & $0.384(0.009-1.543)$ & 0.0254 & $\mathrm{NS}$ \\
hsa-miR-204-5p & $1(0.006-3.160)$ & $0.297(0.006-1.599)$ & 0.0128 & \\
\hline
\end{tabular}

Relative miRNA expression levels for intermediate-risk PTCs are reported as means (minimum-maximum) and normalized to those for

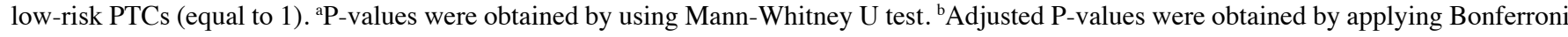
correction. 
miR-146b-5p

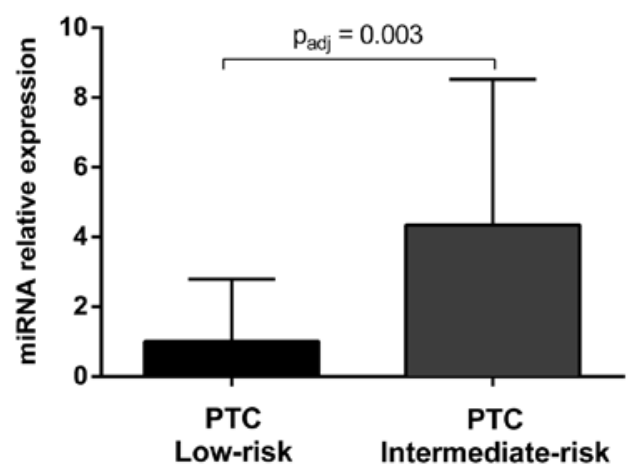

miR-222-3p

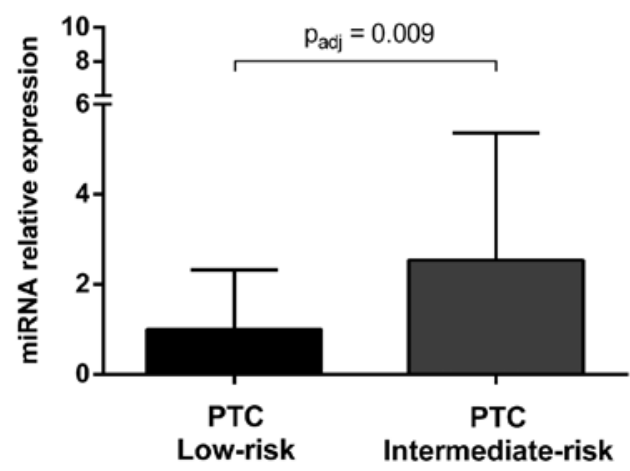

Figure 5. miRNAs associated with risk of recurrence. Expression levels of miR-146b-5p and miR-222-3p are significantly higher in intermediate-risk PTCs than in low-risk PTCs from validation cohort. miRNA levels are reported as mean \pm SD normalized to mean expression levels in low-risk PTCs. P-values were obtained by using Mann-Whitney U test followed by Bonferroni correction.

(miR-146b-5p, miR-146b-3p and miR-204-5p) displayed expression levels in PTCs-FV that were significantly different from those observed in both PTC-CT and PTC-TCV (Fig. 4A). Of note, all three of these miRNAs were expressed in PTC-FV at levels similar to those found in normal thyroid tissue (Fig. 4A).

Subclassification of PTC-FV into encapsulated (PTC-EFV) and infiltrative (PTC-IFV) forms showed that the expression of miR-204-5p in PTC-EFV was similar to that of normal tissue, whereas lower levels were found in all PTC variants, including PTC-IFV (Fig. 4B).

miRNAs associated with risk of tumor recurrence. The 30 miRNAs selected for the validation analysis were further analyzed in low-risk PTCs and intermediate-risk PTCs and a signature of nine miRNAs (i.e., miR-146b-5p, miR-21-5p, miR-222-3p, miR-31-5p, miR-199a-3p/miR-199b-3p, miR-146b-3p, miR-1179, miR-7-2-3p and miR-204-5p) was identified to be associated with a higher risk of tumor recurrence (Table VI). After Bonferroni correction, the expression of miR-146b-5p and miR-222-3p was still significantly upregulated in intermediate-risk PTCs as compared to lowrisk tumors (Fig. 5).

\section{Discussion}

The majority of PTCs display indolent behavior and have an excellent prognosis (2), although certain histological subtypes of PTC are associated with aggressive clinicopathological features and poor outcomes $(3,4)$. Risk stratification is essential to avoid overtreatment of the indolent forms and to provide adequate management for the rare aggressive variants. However, reliable biomarkers for this purpose are currently lacking. MicroRNA expression is frequently dysregulated in cancer cells (9). The high stability of microRNAs in paraffinembedded tissues (10) and body fluids (11) makes them excellent candidates as biomarkers for many cancers, including PTC $(5,12)$. In the present study, we identified an 11-miRNA signature for PTC (miR-146b-5p, miR-146b-3p, miR-221-3p, miR-222-5p, miR-222-3p, miR-1179, miR-486-5p, miR-204-5p, miR-7-2-3p, miR-144-5p and miR-140-3p) (Fig. 2), and two of the 11 (miR-146b-5p and miR-222-3p) were also significantly associated with an increased risk of recurrence (Fig. 5). Overall, these findings confirm the results obtained in earlier studies (5,29-31), as the downregulation of miR-1179 and miR-7-2-3p which were only marginally reported in literature $(32,33)$. The 11 miRNAs mentioned above could be further investigated as diagnostic and prognostic tools for improving the accuracy of preoperative diagnosis of PTC, which currently results indeterminate in $\leq 20 \%$ of cases (19), and for informing decisions on the extent of surgery.

Differential diagnosis of PTC histological variants is also an important challenge since they differ considerably in terms of genetic background, prognosis, and response to surgical and medical treatment (5). To identify miRNAs capable of discriminating between the main histological variants of PTC, we analyzed the expression of 30 selected miRNAs in 74 PTCs from the validation cohort, which included 47 PTC-CT, 20 PTC-FV, and 7 PTC-TCV. In addition, since the prognosis of PTC-FV varies considerably depending on whether the tumor is completely encapsulated or infiltrative (34), we also explored miRNA expression in these two PTC-FV subgroups (PTC-EFV, n=14, PTC-IFV, n=6). We found that the expression of miR-146b-5p and miR-146b-3p was upregulated in both PTC-CT and PTC-TCV, whereas their levels in PTC-FV (both encapsulated and infiltrative subtypes) were similar to those found in normal thyroid tissues (Fig. 4A). As for miR-204-5p, it was downregulated with respect to normal tissue in all PTC histotypes except PTC-EFV (Fig. 4B). In pairwise comparisons, miR-204-5p expression displayed no significant differences between PTC-IFV and normal tissue or between PTC-IFV and PTC-EFV. However, the possibility that miR-204-5p expression is selectively downregulated in the infiltrative subtype of PTC-FV warrants further investigation because this miRNA could be a promising and independent predictor of capsular invasion in PTC-FV. The fact that miR-21-5p was significantly upregulated only in the tall-cell variant PTC is also of interest since this miR might be used as a potential tool for improving the differential diagnosis of this aggressive but under-diagnosed PTC variant (8). The differential expression of miR-146b-5p, miR-146b-3p, and miR-21-5p has been reported in the main PTC histotypes (i.e., PTC-CT, 
PTC-FV and PTC-TCV) (35). As for the FV subtypes, the PTC-FV studied by Sheu and coworkers were all encapsulated tumors. Recently, however, Borrelli et al (36) have identified a miRNA signature that distinguishes encapsulated and infiltrative forms of PTC-FV, although downregulation of miR-204-5p expression in PTC-IFV was not one of the discriminating components of this signature.

In conclusion, this study provides new insights into the molecular underpinning of PTC, highlighting dysregulated expression of several miRNAs that distinguish these cancers from normal thyroid tissue and in some cases display intriguing associations with clinicopathological features of PTCs. The subcohorts of PTCs defined by histotype were admittedly small, especially those of PTC-TCV, PTC-EFV, and PTC-IFV. These preliminary findings need to be confirmed by studies on larger cohorts, which might also reveal additional miRNAs that are differentially expressed in PTC variants. It is also important to recall that all of our experiments were conducted on thyroid tissues from patients who underwent total thyroidectomy. Therefore, to assess their actual value in the context of preoperative diagnosis, our findings will need to be validated in fine needle aspirates from patients with PTCs.

\section{Acknowledgements}

This study was supported by the Umberto Di Mario Foundation, the 'Sapienza' University of Rome (grants C26A13C8C7 and C26A15Z4JM to C.D.). G.G., L.L. and V.P. contributed to this report as recipient of the $\mathrm{PhD}$ program of Biotechnologies and Clinical Medicine of the University of Rome, Sapienza. Writing support was provided by Marian Everett Kent, BSN (European Medical Writers Association) and funded by the Umberto Di Mario Foundation.

\section{References}

1. Davies L and Welch HG: Current thyroid cancer trends in the United States. JAMA Otolaryngol Head Neck Surg 140: 317-322, 2014.

2. Hay ID, Thompson GB, Grant CS, Bergstralh EJ, Dvorak CE, Gorman CA, Maurer MS, McIver B, Mullan BP, Oberg AL, et al: Papillary thyroid carcinoma managed at the Mayo Clinic during six decades (1940-1999): Temporal trends in initial therapy and long-term outcome in 2444 consecutively treated patients. World J Surg 26: 879-885, 2002

3. Lam AK-Y, Lo C-Y and Lam KS-L: Papillary carcinoma of thyroid: A 30-yr clinicopathological review of the histological variants. Endocr Pathol 16: 323-330, 2005.

4. Shi X, Liu R, Basolo F, Giannini R, Shen X, Teng D, Guan H, Shan Z, Teng W, Musholt TJ, et al: Differential clinicopathological risk and prognosis of major papillary thyroid cancer variants. J Clin Endocrinol Metab 101: 264-274, 2016.

5. Agrawal N, Akbani R, Aksoy BA, Ally A, Arachchi H, Asa SL, Auman JT, Balasundaram M, Balu S, Baylin SB, et al; Cancer Genome Atlas Research Network: Integrated genomic characterization of papillary thyroid carcinoma. Cell 159: 676-690, 2014.

6. Lawrence MS, Stojanov P, Polak P, Kryukov GV, Cibulskis K, Sivachenko A, Carter SL, Stewart C, Mermel CH, Roberts SA, et al: Mutational heterogeneity in cancer and the search for new cancer-associated genes. Nature 499: 214-218, 2013.

7. Elsheikh TM, Asa SL, Chan JKC, DeLellis RA, Heffess CS, LiVolsi VA and Wenig BM: Interobserver and intraobserver variation among experts in the diagnosis of thyroid follicular lesions with borderline nuclear features of papillary carcinoma. Am J Clin Pathol 130: 736-744, 2008.

8. Ghossein R and Livolsi VA: Papillary thyroid carcinoma tall cell variant. Thyroid 18: 1179-1181, 2008.
9. Lu J, Getz G, Miska EA, Alvarez-Saavedra E, Lamb J, Peck D, Sweet-Cordero A, Ebert BL, Mak RH, Ferrando AA, et al: MicroRNA expression profiles classify human cancers. Nature 435: 834-838, 2005

10. Hall JS, Taylor J, Valentine HR, Irlam JJ, Eustace A, Hoskin PJ, Miller CJ and West CM: Enhanced stability of microRNA expression facilitates classification of FFPE tumour samples exhibiting near total mRNA degradation. Br J Cancer 107: 684-694, 2012.

11. Mitchell PS, Parkin RK, Kroh EM, Fritz BR, Wyman SK, Pogosova-Agadjanyan EL, Peterson A, Noteboom J, O'Briant KC, Allen A, et al: Circulating microRNAs as stable blood-based markers for cancer detection. Proc Natl Acad Sci USA 105: 10513-10518, 2008.

12. Rosignolo F, Sponziello M, Giacomelli L, Russo D, Pecce V, Biffoni M, Bellantone R, Lombardi CP, Lamartina L, Grani G, et al: Identification of thyroid-associated serum microRNA profiles and their potential use in thyroid cancer follow-up. J Endocr Soc 1: 3-13, 2017.

13. Saiselet M, Pita JM, Augenlicht A, Dom G, Tarabichi M, Fimereli D, Dumont JE, Detours V and Maenhaut C: miRNA expression and function in thyroid carcinomas: A comparative and critical analysis and a model for other cancers. Oncotarget 7: 52475-52492, 2016

14. Swierniak M, Wojcicka A, Czetwertynska M, Stachlewska E, Maciag M, Wiechno W, Gornicka B, Bogdanska M, Koperski L, de la Chapelle A, et al: In-depth characterization of the microRNA transcriptome in normal thyroid and papillary thyroid carcinoma. J Clin Endocrinol Metab 98: E1401-E1409, 2013.

15. Huang Y, Liao D, Pan L, Ye R, Li X, Wang S, Ye C and Chen L: Expressions of miRNAs in papillary thyroid carcinoma and their associations with the BRAFV600E mutation. Eur J Endocrinol 168: 675-681, 2013.

16. Hu Y, Wang H, Chen E, Xu Z, Chen B and Lu G: Candidate microRNAs as biomarkers of thyroid carcinoma: A systematic review, meta-analysis, and experimental validation. Cancer Med 5: 2602-2614, 2016.

17. Aragon Han P, Weng C-H, Khawaja HT, Nagarajan N, Schneider EB, Umbricht CB, Witwer KW and Zeiger MA: MicroRNA expression and association with clinicopathologic features in papillary thyroid cancer: A systematic review. Thyroid 25: $1322-1329,2015$

18. Dettmer M, Perren A, Moch H, Komminoth P, Nikiforov YE and Nikiforova MN: Comprehensive MicroRNA expression profiling identifies novel markers in follicular variant of papillary thyroid carcinoma. Thyroid 23: 1383-1389, 2013.

19. Haugen BR, Alexander EK, Bible KC, Doherty GM, Mandel SJ, Nikiforov YE, Pacini F, Randolph GW, Sawka AM, Schlumberger M, et al: 2015 American Thyroid Association management guidelines for adult patients with thyroid nodules and differentiated thyroid cancer: The American Thyroid Association guidelines task force on thyroid nodules and differentiated thyroid cancer. Thyroid 26: 1-133, 2016.

20. Rosignolo F, Maggisano V, Sponziello M, Celano M, Di Gioia CR, D'Agostino M, Giacomelli L, Verrienti A, Dima M, Pecce $\mathrm{V}$, et al: Reduced expression of THR $\beta$ in papillary thyroid carcinomas: Relationship with BRAF mutation, aggressiveness and miR expression. J Endocrinol Invest 38: 1283-1289, 2015.

21. Sponziello M, Lavarone E, Pegolo E, Di Loreto C, Puppin C, Russo MA, Bruno R, Filetti S, Durante C, Russo D, et al: Molecular differences between human thyroid follicular adenoma and carcinoma revealed by analysis of a murine model of thyroid cancer. Endocrinology 154: 3043-3053, 2013.

22. R Development Core Team: R: A language and environment for statistical computing. R Foundation for Statistical Computing, Vienna. URL http://www.R-project.org/.

23. Wright SP: Adjusted P-values for simultaneous inference. Biometrics 48: 1005-1013, 1992.

24. Pencheva N, Tran H, Buss C, Huh D, Drobnjak M, Busam K and Tavazoie SF: Convergent multi-miRNA targeting of ApoE drives LRP1/LRP8-dependent melanoma metastasis and angiogenesis. Cell 151: 1068-1082, 2012.

25. Dong W, Yao C, Teng X, Chai J, Yang X and Li B: MiR-140-3p suppressed cell growth and invasion by downregulating the expression of ATP8A1 in non-small cell lung cancer. Tumour Biol 37: 2973-2985, 2016.

26. Liu R, Liu C, Zhang D, Liu B, Chen X, Rycaj K, Jeter C, Calhoun-Davis T, Li Y, Yang T, et al: miR-199a-3p targets stemness-related and mitogenic signaling pathways to suppress the expansion and tumorigenic capabilities of prostate cancer stem cells. Oncotarget 7: 56628-56642, 2016. 
27. Kinose Y, Sawada K, Nakamura K, Sawada I, Toda A Nakatsuka E, Hashimoto K, Mabuchi S, Takahashi K, Kurachi H, et al: The hypoxia-related microRNA miR-199a-3p displays tumor suppressor functions in ovarian carcinoma. Oncotarget 6: 11342-11356, 2015.

28. Minna E, Romeo P, De Cecco L, Dugo M, Cassinelli G, Pilotti S, Degl'Innocenti D, Lanzi C, Casalini P, Pierotti MA, et al: miR-199a-3p displays tumor suppressor functions in papillary thyroid carcinoma. Oncotarget 5: 2513-2528, 2014.

29. Chou C-K, Chen R-F, Chou F-F, Chang HW, Chen YJ, Lee YF, Yang KD, Cheng JT, Huang CC and Liu RT: miR-146b is highly expressed in adult papillary thyroid carcinomas with high risk features including extrathyroidal invasion and the BRAF (V600E) mutation. Thyroid 20: 489-494, 2010.

30. Mardente S, Mari E, Consorti F, Di Gioia C, Negri R, Etna M, Zicari A and Antonaci A: HMGB1 induces the overexpression of miR-222 and miR-221 and increases growth and motility in papillary thyroid cancer cells. Oncol Rep 28: 2285-2289, 2012.

31. Qiu YH, Wei YP, Shen NJ, Wang ZC, Kan T, Yu WL, Yi B and Zhang YJ: miR-204 inhibits epithelial to mesenchymal transition by targeting slug in intrahepatic cholangiocarcinoma cells. Cell Physiol Biochem 32: 1331-1341, 2013.

32. Mancikova V, Castelblanco E, Pineiro-Yanez E, Perales-Paton J, de Cubas AA, Inglada-Perez L, Matias-Guiu X, Capel I, Bella M, Lerma E, et al: MicroRNA deep-sequencing reveals master regulators of follicular and papillary thyroid tumors. Mod Pathol 28: 748-757, 2015.
33. Saiselet M, Gacquer D, Spinette A, Craciun L, DecaussinPetrucci M, Andry G, Detours V and Maenhaut C: New global analysis of the microRNA transcriptome of primary tumors and lymph node metastases of papillary thyroid cancer. BMC Genomics 16: 828, 2015.

34. Nikiforov YE, Seethala RR, Tallini G, Baloch ZW, Basolo F, Thompson LD, Barletta JA, Wenig BM, Al Ghuzlan A, Kakudo K, et al: Nomenclature revision for encapsulated follicular variant of papillary thyroid carcinoma: A paradigm shift to reduce overtreatment of indolent tumors. JAMA Oncol 2: 1023-1029, 2016.

35. Sheu S-Y, Grabellus F, Schwertheim S, Worm K, BroeckerPreuss M and Schmid KW: Differential miRNA expression profiles in variants of papillary thyroid carcinoma and encapsulated follicular thyroid tumours. Br J Cancer 102: 376-382, 2010.

36. Borrelli N, Denaro M, Ugolini C, Poma AM, Miccoli M, Vitti P, Miccoli P and Basolo F: miRNA expression profiling of 'noninvasive follicular thyroid neoplasms with papillary-like nuclear features' compared with adenomas and infiltrative follicular variants of papillary thyroid carcinomas. Mod Pathol 30: 39-51, 2017. 